\title{
Bringing reality in physics: System engineering approach to optical phenomena following Huygens' Principle
}

\section{Chandrasekhar Roychoudhuri, Negussie Tirfessa}

Chandrasekhar Roychoudhuri, Negussie Tirfessa, "Bringing reality in physics: System engineering approach to optical phenomena following Huygens' Principle," Proc. SPIE 11143, Fifteenth Conference on Education and Training in Optics and Photonics: ETOP 2019, 111433A (2 July 2019); doi: $10.1117 / 12.2523602$ 


\title{
Bringing reality in physics: System engineering approach to optical phenomena following Huygens’ Principle
}

\author{
ChandraSekahar Roychoudhuri ${ }^{\mathrm{a}}$ and Negussie Tirfessa ${ }^{\mathrm{b}}$ \\ ${ }^{a}$ Physics Department, University of Connecticut, Storrs, CT, USA \\ ${ }^{b}$ Department of Physical Sciences, Manchester Community College, Manchester, CT, USA
}

\begin{abstract}
Optical science and engineering have been playing key enabling roles behind the continuous advancement of physics since ancient times. We have to pick up that role again. Recent publications by physicists Smolin [1], "Trouble with Physics”, 2007 \& Hossenfelder [2], “Lost in Math”, 2018, are deeply troubling for the future of Physics \& our credibility as the gatekeeper of the enabling knowledge of Optical Physics. We will explain how the various erroneous interpretations related to Relativity (e.g. time dilation), to Quantum Mechanics (Superposition Principle) \& Astrophysics (Holographic Universe) arise due to the (i) use of non-causal Fourier modes (existing in all space) as tools of nature's reality and (ii) neglect of Non-Interaction of Waves (NIW). NIW indicates that simple superposition of Fourier modes, in the absence of interacting materials, cannot generate spatio-temporal re-grouping of wave energies. System engineering thinking will be underscored that can constrain our math models to stick to realities. We will present examples on how understanding NIW in optical phenomena can help us bring out causal interpretations and causal models in some of the key branches of Physics.
\end{abstract}

Keywords: Roles of optical scientists in physics; Causal Physics; Non-Interaction of Waves; NIW; System engineering thinking; Quantum Physics; Relativity Physics; Astrophysics

\section{INTRODUCTION}

Optical science and engineering played the key roles during 1600 and 1800 in developing Classical Physics including Astrophysics, and then guided the emergence of Relativity Physics and Quantum Physics through the 1900's. However, starting about the middle of 1900, Optics veered out more as an applied field, leaving the development of fundamental physics in the hands of theoretical physicists dealing with Relativity and QM. These fields are now telling nature how she ought to behave, rather than humbly trying to discover how she actually works [1,2]. Optical scientists have also failed to recognize explicitly a critical property of waves. This is Non-Interaction of Waves (NIW) [3], underscored by Huygens by 1690 [4], even though Fresnel (1817) [5] has mathematically accommodated NIW into the Huygens-Fresnel diffraction integral; again without calling it out as such. NIW automatically underscores the non-physicality of summing infinite Fourier modes to obtain finite spatio-temporal energy pulse in the absence of interacting materials. We also fail to differentiate between un-measurable, mathematically linear, Superposition Principle (SP) from the measurable nonlinear (square modulus) Superposition Effect (SE), displayed by detectors as their physical transformation after the absorption of energy out of all the stimulating waves, not just one wave, as our math correctly indicates. The author explained these in his book, Causal Physics, Taylor \& Francis, 2014 [3]. Many misinterpretations in QM and Relativity can be traced to our conceptual failures summarized above. Better guidance comes from causal system engineering thinking in re-interpreting major optical phenomena. This approach resolves many non-causal interpretations used by physicists in QM (single-photon interference, entanglement, etc.), like our predecessor scientists of 1600 through 1800, optical scientists and engineers should again pick up the leading roles in re-formulating and re-directing the evolution of physics to discover nature's objective reality to assure our sustainable evolution.

Here is one simple example to illustrate that we are getting lost in the beauty and elegance of math [2]. Consider the Gaussian curve. It is one of the most elegant, symmetric and beautiful mathematical expression, which is used very effectively for quantitative representation of diverse characteristics in almost all branches of human endeavor, not just in physics and engineering. The vertical axis of a Gaussian curve usually represents the quantitative variation of a physical attribute, which varies with interdependent variable parameter represented in the horizontal axis. Mathematically, this Gaussian curve extends to infinity in both the positive and negative horizontal directions. However, no real physical attribute, physical, biological or sociological parameter, can have infinitely large values. Thus, the elegant and beautiful Gaussian representation of physical reality is no more than approximate, albeit a very good one. All physical attributes 
must always have finite quantitative values. Thus, the real "Gaussian" curve must terminate to zeros on both the sides of the horizontal axis. However, being lost in the elegance of math, we never encourage students to explore as to (i) why and (ii) how, the Gaussian attribute terminates to zeros. If the students are asked to explore potential realities behind the actual termination of the Gaussian curve to zeros, they will discover new knowledge, new science, which we have been neglecting to explore. Consider an examples from optical physics. The mathematical solution to the fundamental transverse longitudinal mode-pattern from a well-designed laser cavity is a Gaussian. The quantitative measurement also shows it to be essentially "Gaussian", although we terminate the curve to zero as a very good approximation. However, we fail to explore the physical sciences behind this very good approximate match. The transverse size of any laser-gain medium usually abruptly terminates to zero at the boundaries. So, the Gaussian mode inside the cavity is also bound to terminate to zero. Have we explored how nature manages to accept "zero boundary values", while our elegant mathematical model represents it with zeros that has to go to infinity? Are we depriving ourselves from learning anything further about the microscopic physical processes going on in nature?

The interested readers should note that this article is trying to promote the concept of re-building physics using fundamentally new way of thinking, which is an extremely difficult task. Therefore, the author apologizes that this article may appear to be a bit rambling. However, there are serious ideas and concepts in this paper, which the author has developed as an experimental optical physicist over the last fifty years.

\section{BACKGROUND OF THINKING BEHIND STRUCTURING THIS PAPER}

\subsection{Background}

Unification of theories: The history of advancement in science underscores that consistent and iterative efforts to integrate diverse theories lead to better understanding of the rules of operations going on in nature. Newton integrated the rules of classical mechanics by integrating inertia, the inverse-square law of gravity and the acceleration due to gravity. Maxwell integrated Electrostatics and Magnetostatics into Electromagnetism. These theories are still remaining essentially unchallenged in our modern world. After developing the Special and the General theories of Relativity, Einstein spent more than a quarter of his last life to develop a unified field theory. In the meantime, the basic mathematical formalism of Quantum Mechanics, or QM (1925-1930) had achieved its undeniable successes. Unfortunately, the physical interpretations of both these theories of Relativity and QM defy common-sense causality and forces us to accept mathematical extra dimensions as added new real dimensions of nature. Prolonged attempts by the global best-and-the-brightest have not been successful to bring about a higher level of integration of these theories to give us the sense that physics is heading in the right direction. Accordingly, the volume of serious dissents and criticisms against current pure physics have been steadily increasing. However, this paper is not focused on criticism. It proposes and defines concrete paths towards new attempts of unification of different physics theories by remaining focused upon experiments, observations and causality.

Broad enabling power of optical sciences: We want to leverage the inherent powers of the fields of optical science and engineering. These fields have been advancing unabated since ancient times without any break. New optical fields of nano-photonics, bio-photonics, plasmonic-photonics, opto-genomics, etc., are thriving based upon postulates and theories developed during 1600's through 1800's. It is also important to recognize that optical science, engineering and measurement tools have been providing the core guidance behind the advancement of most branches of physics since ancient times. The Nobel Committee has awarded a good number of prizes in Physics during the last three decades to people who have been developing/using optical concepts, tools and technologies to advance physics.

Leveraging Huygens Principle: This paper proposes to underscore that we can leverage Huygens' Principle (HP) of optical wave propagation to re-vitalize physics through the unification of existence of EM waves and particles as the excited states of the same universally stationary Cosmic Tension Filed, or CTF (old ether; to be defined later). The enduring success of HP derives from Huygens' insistence on visualizing the real physical processes behind the generation and propagation of waves [4]. We have renamed this approach as belonging to the broad class of Interaction Process Mapping Epistemology, or, IPM-E. The modern epistemology behind the "evidence based physics" has essentially remained focused on Measurable Data Modeling Epistemology, or MDM-E. Mathematics reigns supreme; evidences are sought out to support the theories; as if the invisible ontological interaction processes that generate the data are irrelevant. In this paper, we underscore that the prevailing successes of MDM-E must be strengthened and 
advanced further through the incorporation of IPM-E. This will guide us iteratively to get closer and closer to nature's ontological reality.

Built-in physics behind Huygens' Principle: The core physics behind the success of Huygens' Principle was his recognition that every point on a wavefront (i) serves as the source for generating secondary wavelets, (ii) which keep propagating and evolving forward without interacting with each other. Note the implementation of wave propagation process (or IPM-E) by Huygens. Fresnel's integral representation literally sums the expanding secondary wavelets out of every point of the emanating aperture. They keep diverging and evolving through each other unperturbed by each other's' presence. This is precisely NIW. However, optics books do not call this out explicitly.

Cosmic Tension Field, or CTF, gives birth to both EM waves and elementary particles: For every space-point to be the source for a new wavelet, the "free space" has to be a reservoir of electromagnetic and other forms of energies as a tension field. Perpetual wave propagation, independent of wave-generating source velocity, has to be sustained by a tension field when excited within its linear restoration limit. We are re-naming this field as the Cosmic Tension Field, or CTF [6, Ch.11 in 3]. This is to differentiate the name CTF from the old ether that was rejected based on MichelsonMorley's null ether-drag experiments. We can now introduce a major unification potential in physics if we also postulate that all stable elementary particles are some form of localized, in-phase, self-looped (hence, self-resonant) and vortex- or torus-like oscillations of the same CTF. Then, such torus-like oscillations of the CTF would not drag CTF with it, just like propagating waves and vortices in fluids under tension do not drag the parent tension field. Since material bodies are ultimately assemblies of elementary particles, material bodies would not drag the parent tension field as they move from one spatial point to another. This immediately accommodates the null results of Michelson-Morley experiments. Note that Schrodinger's "wave equation" accepts free particles as harmonic oscillators, exp[-iEt/ $\mathrm{h}]$, or exp[-i2 $\pi \mathrm{ft}]$, where $\mathrm{E}=\mathrm{hf}$. This harmonic time-function should not be interpreted as plane waves! Plane waves, in general, do not exist in nature; as they would violate the law of conservation energy. This is just one important unification step. The chapters on Relativity, Astronomy and Cosmology will demonstrate more examples of unified explanations for inter-related phenomena.

Non-Interaction of Waves (NIW), a major contribution of Huygens in Physics: The NIW-property, first formally identified by Huygens [4], has been systematically ignored, perhaps, because of the fame of Newton's corpuscular model for light and Einstein's “indivisible light quanta”. These concepts have completely over-shadowed Huygens' wave concept. This historic neglect facilitated our failure to appreciate explicitly wide ranges of physical significance in understanding and interpreting many optics related phenomena. We have been consistently ignoring the following theoretical and observed facts. First, (i) light propagates as amplitude undulations of an electric tension gradient $\varepsilon_{0}^{-1}$ opposed by the generation of the magnetic resistance $\mu_{0}$, giving rise to the wave velocity $c^{2}=\varepsilon_{0}^{-1} / \mu_{0}$. This modified expression is meant to bring out the similarity between the wave equations for EM waves in CTF and mechanical waves in material string under mechanical tension, $\mathrm{v}^{2}=T / \sigma$ ( $T$ is the mechanical tension and $\sigma$ is the mass per unit length.). EM waves do not propagate as indivisible light quanta. Otherwise, Huygens-Fresnel diffraction integral (1817) and Maxwell's wave equation (1867) could not have been successfully guiding the fields of optical science and engineering un-interrupted and unabated until today. Second, (ii) linearity of second order differential equation dictates that multiple waves (all solutions of the equation) can propagate through the same physical volume at the same time as long as the sum total amplitude remains within the limit of the Young's modulus of the tension field-strength (restoration of the oscillatory wave amplitudes). This is the mathematical origin of the Superposition Principle (SP) within the linear regime. However, observing (registering) the Superposition Effect (SE) can take place only after an optical detector, resonant to the optical frequency, executes the square modulus operation and absorbs energy from all the superposed waves that are stimulating it simultaneously. This is how our causal mathematical expressions are written always for superposition phenomenon. Quantum Mechanics cannot localize EM waves as localized "indivisible light quanta”. By virtue of the inherent NIW property of waves, the summation of a mathematically correct set of Fourier monochromatic modes cannot materialize as space-localized energy pulse ("photon") in the real world.

Hybrid Photon eliminates the need for the postulate of "wave-particle duality": One of the key point out of these discussions is that we can eliminate the postulate of wave-particle duality that has been muddling the interpretation of quantum physics for almost a century and has been one of the major causes behind the retardation in the progress of physics. Photon exist as a "light quanta" only for a brief instant; then it evolves as a finite wave packet as per HuygensFresnel diffraction integral. This is why optical engineers never need to struggle with quantized "photon". 
Enduring power of Huygens Principle: We can further appreciate the enduring significance of Huygens' Principle from the following observations. Even though Newton's Law of Gravity is sufficient in planning and tracking the trajectories of satellites sent all the way to Pluto and beyond; it fails to explain the velocity distribution of stars from the center of a galaxy, measured, so far, for a couple of hundred galaxies. Even Einstein's General Relativity has not been of much help here. This has triggered the formulation of the postulates, Dark Energy and Dark Matter. However, for over twenty years of intense theoretical and experimental research, we are far from establishing the reality of Dark Matter and Dark Energy. In contrast, first, Huygens Principle, proposed and used for the broad field of optics since 1670, has remained valid in all applied cases. Second, with our proposed extension of Huygens ether as CTF to accommodate the emergence of particles as its excited states, one does not need the postulates of Dark Energy and Dark Matter. Hundred percent of the energy of the universe is always contained by the CTF as the sum of its dormant and excited states (EM waves and "material" particles). This is the causal root behind the universal law of conservation of energy. The various excited states of the CTF, waves and particles, can interact with each other and only undergo physical transformations conserving the total energy. However, the entire observable universe remains as different forms of excitations of the stationary CTF. Therefore, CTF also serves as the stationary universal reference frame in which cosmic evolution is playing out.

\subsection{Logically structuring our thinking}

We have structured our thinking as presented in the chart (Fig.1). The content of the two boxes, 2-3, immediately below the top box, 1, underscores the visualization of the physical processes behind wave propagation. The general observation is that all waves propagate spreading out diffractively. Huygens pictured that, each point on the wavefront acts as a new

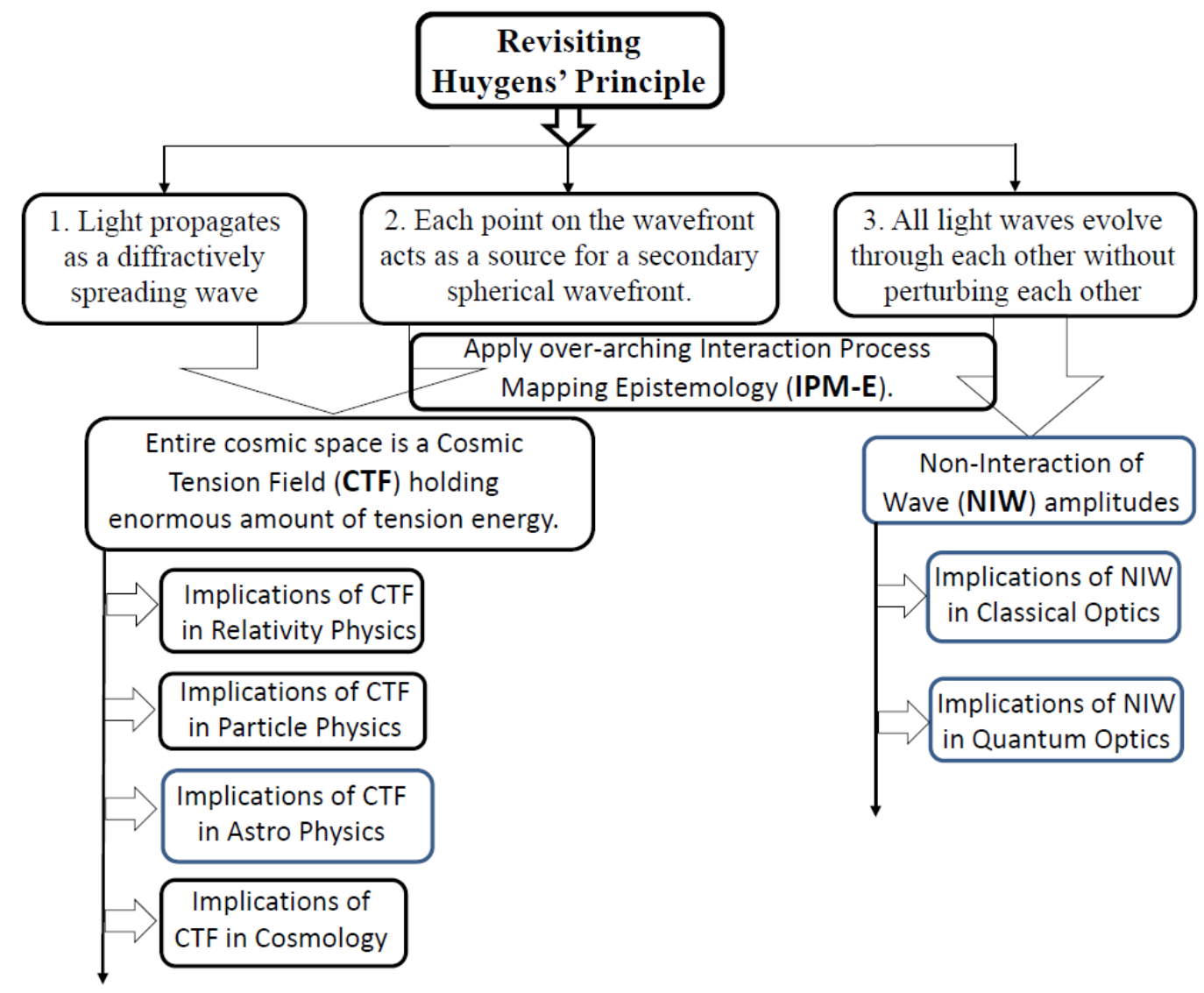

Fig.1. Logic-Chart. Huygens process driven thinking to model light propagation has given us (i) the NonInteraction of Waves and (ii) the concept of the Complex Tension Field (CTF), which together provides us with logical unification tools to re-formulate physics out of one single cosmic field. 
source point for the generation of a new (secondary) wavelet, which can conceptually explain the observed spreading of waves as they propagate. However, Huygens needed another postulate, the Non-Interaction of Waves (NIW-property) between the secondary wavelets, to assure that the secondary wavelets do not spatially break up each other's wave energy distribution as they keep propagating forward. The author has generalized Huygens' NIW-concept to all waves under linear environment [3]. If all the points on any propagating wave can act as a source for secondary wavelets, then it is obvious that Huygens was intuitively visualizing an energetic tension field permeating all space holding a uniform distribution of dominantly electromagnetic energy. The oscillations of the electric tension and magnetic resistance are generating and sustaining the perpetual propagation of light waves. Of course, this is precisely the mathematical model which was given much later by Maxwell's wave equation in 1867, expressing the light velocity, $c^{2}=\left(1 / \epsilon_{0} \mu_{0}\right)$, where $\epsilon_{0}$ and $\mu_{0}$ are the electric tension and magnetic resistance held by the all-pervading "ether".

The narrow elongated horizontal box-4 in the third line, underscores the thinking model of early scientists that we must strive to understand and try to visualize the physical interaction processes that nature executes to bring about material changes behind the persistent observable evolutions in the universe. We are calling it out as the Interaction Process Mapping Epistemology, or IPM-E. Throughout the paper, we will use IPM-E over and above the prevailing successful mode of thinking, the Measurable Data Modeling Epistemology, or MDM-E. MDM-E driven modern "evidence based science" has become quite stagnant for some time now at the level of fundamental physics [1,2]. That is why we are underscoring the need for re-introducing the incorporation of IPM-E along with MDM-E, based on our recent successes [3]. Once we combine the boxes $2 \& 3$ with the IPM-E box-4, we see that the lingering concept of ether since ancient time must be brought back. We need to anchor our mathematical models to reality by simultaneously modeling the invisible physical interaction processes that give rise to the measurable data. Let us recognize here that experiments demonstrating the null ether-drag by Michelson-Morley cannot refute the existence of the ether-like medium permeating all cosmic space. As designed, their experiments only refute the anticipated drag of "ether" by material bodies [6-10].

Therefore, the knowledge and epistemology behind the boxes 2, 3 and 4 give rise to three new categories of discussions to be summarized in this paper. They are identified as CTF (Cosmic Tension Fields), NIW (Non-Interaction of Waves) and IPM-E \& Math. The columns depicted by CTF and NIW represent the two core sections of this paper. IPM-E \& Math section will deal with the limitations of math and justify the incorporation of visualizing the invisible interaction processes in nature. Even though this is counter to the Copenhagen Philosophy, the sustainable human evolution and the necessary technology development will require us to emulate system-engineering feats that nature has been executing. The key purpose of this paper is to demonstrate that the acceptance of NIW and CTF, empowered by IPM-E, makes physics exciting again. This new thinking opens up many new possibilities for open-ended investigations and innovations, rather than remaining bound by the foundations laid by the theories of Relativities and Quantum Mechanics. The concept of CTF, or its future enhanced version, can provide us with the unifying physical field that Einstein was looking for. The unifying filed must have the key fundamental properties of giving rise to both the electromagnetic energy as perpetually propagating waves and the elementary particles as different kinds of localized, self-resonant vortex-like (or torus-like) oscillations. Translation of material bodies, assembly of such vortices, will certainly not drag the parent CTF fluid. Schrodinger's quantum mechanical wave equation already indicates this mode of behavior of particles. Schrodinger's key expression for a free-particle is $\exp [i E t / \hbar]=\exp [i 2 \pi f t]$, where $E=h f_{\text {in }}$. This exponential relation does not represent a "Plane Wave" or a "Pilot Wave". " $\mathrm{f}_{\mathrm{in}}$ " is the intrinsic and characteristic energetic harmonic oscillation frequency of the localized self-resonant (closed-looped and in-phase) torus-like oscillations. The appropriate mathematical model will have to be developed to represent such torus-like oscillations of CTF. The in-phase resonant oscillations are at the root behind the emergence of quantized energy levels (quantumness) in the micro world of nuclei, atoms and molecules.

In the following sections, we will summarize the sub-contents identified under the three boxes, 5, 6 \& 7 shown in Fig.1.

\section{PHYSICS GUIDED BY THE CONCEPT OF A COMPLEX TENSION FIELD (CTF)}

\subsection{Relativity Physics in light of CTF}

This section underscores that the two key postulates, presented by Einstein in his Special Relativity [11], emerges logically out of the existence of CTF as the sustainer of both the propagating EM waves and the localized oscillating "particles" across the entire universe. The velocity of light will be the same everywhere in the universe since the tension properties $\epsilon_{0}$ and $\mu_{0}$ are same everywhere in the universe contained by the stationary CTF. When a material medium 
(assembly of CTF-torus's) intercepts the EM waves, its velocity reduces due to the reduced bulk electromagnetic tension property of the assembly of the particles. The velocity of the "trapped" EM waves suffers "Fresnel Drag" when the bulkbody moves through the CTF. Einstein's second postulate posits that the laws of physics are same everywhere in the universe. This also emerges logically since the waves and the particles are emergent excited states of the same stationary CTF all across the universe.

Running time cannot be physically dilated, because no physical bodies possess a parameter directly related to the running time. We measure characteristic frequencies of various oscillating entities. We create a secondary parameter, a time interval or a period, by inverting the frequency. Therefore, by changing the physical environment around the oscillator, its characteristic frequency can be dilated or contracted. Twin Paradox is a distracting mathematical debate only. We should also note that it is rather rare to find material bodies or molecules in nature, in stars, in cosmic clouds that move with velocities very close to that of the light except in human constructed giant accelerators.

CTF naturally accommodates Einstein's core concept of gravity as the "Curvature of Space”, which can be imagined as stable potential gradients in the CTF around assemblies of oscillating particles (stars, etc.). If a large body of localized mass suddenly explodes into a thinly spread out dust and molecules, the extended potential gradient will take a finite time to collapse into some very weak and distributed "curvature".

\subsection{Particle Physics in light of CTF}

As mentioned earlier, we can accommodate the absence of ether drag, established by Michelson-Morley experiment, by proposing that material bodies are assemblies of self-looped torus-like oscillations of the same CTF [3.6]. Translation of assemblies of such torus-like oscillations through the stationary CTF would not cause any drag of the CTF. CTF itself remains stationary. We now need to first develop and strengthen the foundational properties of the CTF through various other existing and new experiments that will assure us that the basic postulate that particles are localized self-looped oscillations of CTF. The emergence of various forces have to be explained as different kinds of averaged potential gradients around different kinds of self-looped oscillations. The origin of forces in the CTF model cannot be explained via exchange-particles of various kinds.

Particle superposition experiment, as CTF oscillators, have been modeled by using our localized harmonic oscillation model [12]. Again, the non-causal postulate of wave-particle duality is not necessary to explain Superposition Effects (SE) due to particle beams. Since ancient times "duality" in physics implied ignorance behind the phenomenon under discussion. This was true for wave-particle duality that originated with Newton (corpuscular) and Huygens (wavelets) during 1670's. Both acknowledged that the dual models were perceived because of the-then ignorance regarding the deeper physical reality about light waves. Maxwell derived the solution for the "ether wave" much later in 1876. However, for lack of our ability to model particles as oscillation of the modern ether as CTF, we keep on using waveparticle duality as our temporary knowledge.

\subsection{Astrophysics and Cosmology in light of CTF}

\subsubsection{Doppler Effect}

CTF represents the "preferred and stationary reference frame" for our universe, like Lorentz-ether. Since all atoms and molecules are diverse assemblies of various torus of this CTF, any emerging light pulse in the CTF will suffer Doppler frequency shift due to the "absolute" source velocity with respect to the stationary CTF-frame. This light pulse will then keep propagating with the velocity "c" across the entire universe, preserving its Doppler shifted frequency. The original quantum transition frequency is now modified into a permanent and real Doppler frequency shift due to the source-only movement. If a set of detecting atoms with different relative velocities w.r.t. CTF, receive this Doppler shifted wave, the perceived frequencies will be different for different atoms [see Ch.10 in 3]. The original Doppler Effect was expressed this way. We use this original formalism due to Doppler when we analyze frequencies of waves generated in the tension fields of material media like pressure tension field (sound waves), surface tension field (water waves), etc. It should not be any different for EM waves propagating as oscillation of CTF.

\subsubsection{Cosmological Redshift}

The above discussion automatically leads us to the Cosmological Redshift originally discovered by Hubble. Is it really due to Doppler Effect, or some other physical phenomenon? This controversy already exists in the literature. We believe this shift is due to very slow, linear distance dependent energy loss of all EM waves as they propagate through cosmic "cloud" permeated CTF [8]. 


\subsubsection{CMBR}

The Cosmological Microwave Background is another observed fact. However, acceptance of the stationary CTF and the unchanging rules of operation behind the interaction processes in the cosmic system faces logical contradictions with the hypothesis of expanding universe. Expanding universe implies that the field CTF is expanding and, consequently, its intrinsic tension values $\varepsilon_{0} \& \mu_{0}$, which determine all the intrinsic characteristics of particles and the fixed velocity of EM waves, would also have been changing with time. Note that particle rest mass $m_{0}=E / c^{2}=E \varepsilon_{0} \mu_{0}$ should also change if space is expanding. There are no such validating observations. Further, this line of argument that the enormous expansion of the early-epoch-space has dilated the wavelength of the EM waves has at least one more serious problem. In the quantum world, in the atomic and molecular emissions, the frequency is the primary physical parameter dictated by the quantum transition characteristics. This original quantum frequency of light remains unchanged as it propagates through different media of different refractive indices, even though the wavelength changes. We believe that CMBR is a pure simple Blackbody Radiation, which is in thermal equilibrium with the thinly spread cosmic dust and molecules. In our laboratory Blackbody, the thermal equilibrium is attained due to innumerable scattering and reflections from of EM waves from the molecules of the inner surface of an enclosed "blackbody" surface maintained at some adjustable thermal equilibrium. In the gigantic cosmic space, the thermal equilibrium is established due to innumerable absorption and re-emission of the radiation by the cosmic dust, which are at $2.7^{\circ} \mathrm{K}$. Of course, the stars in galaxies are at much higher temperature. Their coronas also closely follow the rules of Blackbody radiation.

\section{CLASSICAL AND QUANTUM OPTICS IN VIEW OF NIW}

\subsection{Quantum Optics in view of NIW (Non-Interaction of Waves)}

\subsubsection{Measurement Problem (Ensemble Interpretation)}

The founders of QM correctly identified that our measurement has some fundamental problems. One is wave-particle duality and the other is uncertainty. The Copenhagen Interpretation has apparently resolved the problems by accepting the wave-particle duality as our new confirmed knowledge without finding any deeper knowledge behind the structures of photons and electrons. The uncertainty is considered to have been resolved mathematically and by assigning it to nature's inherent mode of operation. Even though this assignment creates consistent causality violation in the micro universe. Again, we have been taking this as our confirmed new knowledge. This is a surprising approach in science because all of our mathematical equation for physics theories are constructed as hard-core causal representation of equating measurable effect with the presumed or hypothesized cause(s). We have been accepting these built-in contradictions as our new mode of thinking. Then the intellectual and collective social skills to interpret elegant equations become the driving force behind the design of new experiments to extract the desired evidences. Naturally, we experience persistent confusion due to the diversity of subjective interpretations we find in books. The way out is to challenge ourselves to visualize the causal (ontological) physical processes that are being executed by nature.

We propose a way out of this dilemma by first recognizing that we cannot overcome the measurement problem by using elegant mathematics and/or interpretations. Second, the data in any instrument emerges as some quantifiable number due to some physical changes in our chosen interactants, none of whose properties is ever known completely by us. Further, we can never claim that our instruments have $100 \%$ fidelity. Thus, the real issue is that we have a perpetual Information Retrieval Problem (IRP), not just a measurement problem. We cannot resolve the issues behind IRP by elegant mathematics alone. We have to continue our hard work iteratively by developing newer theories, while developing more and more refined measuring instruments. The approach will be further empowered by iterative approach to unification of theories, while explicitly paying attention to visualize the invisible interaction processes that give rise to the measurable data. However, during each iteration, we must develop improved and integrative new set of basic postulates that combines all the theories to be unified. Attempts to preserve all the basic postulates of each individual working theories will only generate persistent bottlenecks. Because each working theory is a logically closedsystem to explain a small set of phenomena, which the theory was constructed to explain. Let us appreciate that all working theories are necessarily incomplete, as they are formulated based upon insufficient knowledge of the universe we are trying to model. In this context, theoretically oriented people should ponder on Gödel's Incompleteness Theorem. This is only a brief discussion on the measurement problem. This is to facilitate the discussions on quantum optics related issues in the following section. 


\subsubsection{Hybrid Photon}

Photons as "indivisible light quanta" has been continuing since 1905. It has only contributed to confused interpretations that violate causality. No optical scientist or engineer propagate "indivisible light quanta". They propagate light waves using Huygens-Fresnel diffraction integral (or its different mathematical variations) along with Maxwell's wave equations when the intervening material properties are being modified by the wave. Further, the independent "indivisible light quanta" have never been successfully localized. Yet, the QM formalism correctly models the emission properties of "photons"; and the classical optical-wave propagating formalism correctly predicts the instrumental data. Accordingly, it is necessary to resolve the wave particle duality by defining a hybrid photon.

Spontaneous emission, on classical spectroscopic measurements, show Lorentzian line width, as if light beam out of atomic dipole consists of exponential pulses of a finite temporal duration [see Ch.5 in 3]. Some of the measured spectral line widths are in the domain of $\mathrm{GHz}$, implicating temporal duration of the order of nanoseconds. Doppler-free tunable laser spectrometry has successfully measured $\mathrm{v}_{\mathrm{mn}}$ in the domain of $\mathrm{MHz}$ [13]. These and other observations, give us confidence that at the moment of quantum transition, an atoms or a molecule, does release a quantum of energy given by $E_{m n}=h v_{m n}$. Now to conform to the staggering successes of classical optics and engineering, riding on Huygens principle, it is quite rational to assume that this energy quanta $h v_{m n}$ must immediately evolve into a quasi-exponential classical wave pulse and propagate out diffractively oscillating at the quantum-transition frequency $v_{\mathrm{mn}}$. This model easily accommodates the generation of Doppler frequency shift due to the source velocity and detector velocity with respect to the stationary CTF.

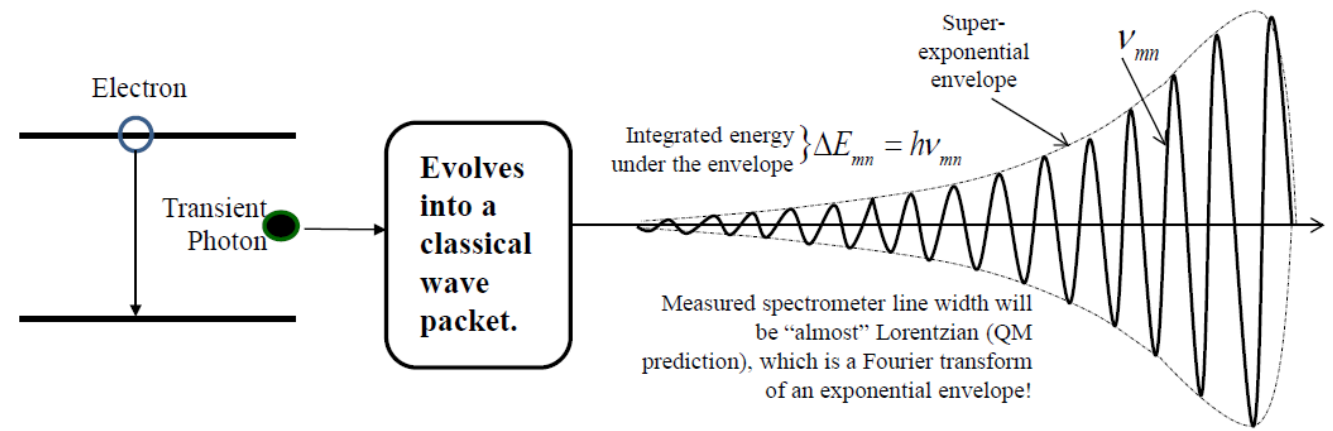

Figure.2. Hybrid photon model. At the instant of emission from atoms or molecules, the photon of energy hv is a quantum entity, which then triggers the emergence of a classical wave packet the same energy with a quasi-exponential temporal envelope and the QM-predicted carrier frequency $v$.

\subsubsection{Superposition Effects with Hybrid Photons without Duality}

This sub-section gives the summary of an experiment carried out by the author [14] using a well-known Mach-Zehnder interferometer (MZ) to demonstrate that superposition effect, to become manifest, require physical superposition of the real physical signals that we write down in the superposition Eq.1. For a two-beam superposition experiment, we have two amplitudes along with their respective phase information. A single stable elementary particle cannot carry these four parameters, as a single quantum photon is claimed to do. A stable elementary particle, by definition, cannot be multivalued at the same moment in the same space. Accordingly, the "indivisible single photon interference" is an erroneous interpretation. When our electronic counter registers a "click", it is a highly amplified current pulse consisting of, most likely, hundreds of millions of electrons, triggered by the release of the original photoelectron, bound quantum mechanically within the assembly of the detector molecules. Discreteness and quantumness lies with the bound electron, not with the EM waves. Further, quantum transitions do not always require a quantized donor. Mechanical energy of striking stones produces spark and light through quantum ionizations and downward transitions. This quantum process was at the root behind the discovery of controlled fire generation by our very distant ancestors. This was much before they invented any language with grammar. We should recognize that mathematical theories did not guide the most critical phase of early human evolution.

Let us get back to our demonstration experiment. We arranged the two equal-energy incident beams in the output beam-combiner of the MZ from the opposite sides in such a way that the Poynting vectors for the two pairs of output beams are exactly coincident and collinear. Then the classical energy conservation demands that all the energy of the two beams will go to only one direction or the other as the relative phase difference between the two beams is arranged to vary from zero to pi. This is a simple and old classical boundary-value problem. There is a pi-phase shift for "external 
reflection" compared to the "internal reflection" from the two sides of the beam combiner. Therefore, for zero or pi relative phase delay, all the energy from both the beams will be directed into one or the other direction.

It is correct that classical light pulses are generated by atoms through quantum processes. The classical boundary conditions of a beam splitter arise as bulk properties out of the assembly of quantum atoms. However, the attempt to develop the novel interpretation of EM waves consisting of "indivisible photons" represents logically inconsistent physics. We should not encourage the introduction of complex and non-causal quantum interpretation just by reducing the photoelectric counts to a very low rate. Light always propagates as diffractive wave packets and follows classical boundary conditions. There is no need for wave-particle duality. The next sub-section explains that even for particle diffraction, the wave-particle duality is unnecessary.

Another major and fundamental interpretational problem behind "single photon interference" lies with the assumption that no energy ("photon") arrives at the dark fringe locations. In reality, at the dark fringe locations, the resultant Evector stimulation of the detecting dipole is zero. Un-stimulated detecting dipole cannot absorb any energy out of the fields passing-by. Surprisingly, our mathematics clearly tells us that the location of dark fringes are defined by the zero resultant electric field value as experienced by the detecting dipoles (see Eq.2). Data can be generated in a detecting instrument only after some physical transformation is experienced by the detecting element (or material). This physical transformation require energy absorption from an incident EM field. For light-matter interaction, the dipolar polarizability $(\chi)$ of the detecting molecule will determine the resultant strength of the stimulation. For multiple incident waves, the Superposition Principle (SP) determines the degree of stimulation (Eq.1) and the measurable Superposition Effect (SE), Eq.2 determines quantity of energy transfer:

$$
\begin{gathered}
\psi=\chi E_{1}+\chi E_{2}=\chi a_{1} e^{i 2 \pi v(t+\tau)}+\chi a_{2} e^{i 2 \pi v t} \\
D(\tau) \equiv \psi^{*} \psi=\left|\chi a_{1} r e^{i 2 \pi v(t+\tau)}+\chi a_{2} t e^{i 2 \pi v t}\right|^{2} \\
=\chi^{2}\left[\left(a_{1}^{2}+a_{2}^{2}\right)+2 a_{1} a_{2} \cos 2 \pi v \tau\right], \text { for narrow frequency light } \\
=2 \chi^{2} a^{2}[1+\cos 2 \pi v \tau]=4 \chi^{2} a^{2} \cos ^{2} \pi v \tau \text {; only when } a_{1}=a_{2}=a
\end{gathered}
$$

In Eq. 2 whenever the factor $2 \pi v \tau$ ( $\tau$ being the relative delay between the two beams) becomes an odd multiple of $\pi$, the detected signal will be zero, and specifically under the condition $a_{1}=a_{2}=a$. This is because the two stimulating signals are out of phase and cancel each other's stimulation on the detecting dipole. The location of the unstimulated detector remains dark. It is not because no signal, or no photon, arrived there. The EM waves simply passes through the detecting molecule without being able to stimulate it and share part of their energy with that detecting element. This the causal interpretation dictated by our causal mathematical equation. We just need to pay close attention to the physical interaction processes that generate data. Eq. $1 \& 2$ are derived heuristically without directly modeling quantum transition, which is embedded in $\chi$. Lamb and Scully [15] has derived proper semi-classical formulation for photoelectric effect.

\subsubsection{Photoelectric Effect due to random superposition of many wave packets}

Einstein derived the photoelectric equation using the simple Measurable Data Modeling Epistemology (MDM-E). It was an energy balancing equation. It did not explicitly model the underlying physical amplitude stimulation of the bound electrons within the material before their release through energy absorption (see Eq.1 \& 2). Electrons are quantized particles and they are always bound quantum mechanically in atoms, molecules and solids. However, this detailed knowledge was not available to Einstein during very early 1900. Einstein's brilliance was in in recognizing the quantumness in the photoelectric data, a cut-off frequency for electron release. He was right in postulating $\Delta \mathrm{E}=\mathrm{hv}$ for bound electrons. However, had he correctly assigned the quantumness to electron, rather than to light waves, he would have invented Quantum Mechanics some twenty years earlier [16]

\subsubsection{Superposition Effects due to particles without duality}

Very similar equations, Eq.1 \& 2, can also be used to explain Superposition Effect due to particle beams. The origin of the harmonic phase oscillations for EM wave is the "waving" or oscillation of the electric vector. However, the particles do not propagate as waves. Their stability derives from their localized self-looped torus-like harmonic oscillations, $\exp \left[i 2 \pi f_{\text {in }} t\right]$, which is also a Schrodinger representation of a free particle. However, the inertial particles stay put unless acted upon by some external force, which is a potential gradient around them, in the CTF, generated by some other 
particles or their assembly nearby. This is why de Broglie introduced his particles possessing some characteristic wavelength, for its kinetic motion $\lambda=h / m v$, without defining what is "waving". This de Broglie relation also has some inherent mathematical problem. This relation becomes divergent when the velocity of the particle becomes zero. However, superposition effect with particle beams is very well established. Accordingly, we need to introduce a different kind of harmonic oscillation of particles $\exp \left[i 2 \pi f_{k t} t\right.$, which is always generated when they are given some kinetic

energy, $h f_{k t}=m \mathrm{v}^{2} / 2$. Use of this exponential phase factor in Eq.1 and 2 will generate the particle superposition effect without conceptual struggle as to what is "waving" with the localized particles. In addition, as far as the "wave-particle duality" is concerned, the same set of arguments, as in sub-section 4.1.3, can be extended that there is no need to invoke duality. Particles are always particles and waves are always waves. Energy exchange between them, or inter-conversions can happen only when they interact.

We are assuming that when we attempt to detect the superposition effect due to particle beams, a normal dipolar quantum detector can become excited and then absorb the energy out of the impinging particles as the square modulus of the non-zero resultant amplitude stimulation and display a "bright fringe". The "dark-fringe" locations are where the sum total resultant induced amplitude stimulation is zero due to multiple particles carrying different kinetic phaseoscillations. We have expressed this same physical model for superposition effects due to light beams having electricvector oscillations. The approach automatically implies that the dark fringe locations do receive the superposed particles arriving, say, from a double-slit set up, but could not stimulate and deliver their energies as they were mutually out of phase with each other and could not stimulate the detecting molecule. The null stimulation leaves the detecting molecules "unexposed". Accordingly, we have proposed several experiments using Rb-atom beam to validate this causal approach to particle superposition effect [3,12]. If one uses a thick $\mathrm{AgBr}$ holographic plate to detect the fringes of superposition, then after the normal development of the plate and then its observation in white light will show the expected dark/bright fringes. However, illumination with a Rb-resonance light beam of $780 \mathrm{~nm}$ would show Gaussianlike distribution of scattered Rb-atoms. Atoms and particles do not diffract like Huygens secondary wavelets, as EM waves do. Once these proposed experiments are validated, we will have a causal model for particle superposition effect consistent with that due to light beams. Neither of them need to use the non-causal model of wave-particle duality.

\subsubsection{Entanglement, Bell's Theorem \& Ensemble Interpretation (in view of hard causality)}

Let us first clarify the broad meaning of the word "entanglement". Our firm position is that the universe is evolving through diverse kinds of interactions between different entities following strictly causal rules of operations; we have been trying to discover. Our successes in understanding most of the observations in the universe through repeated experiments and modeling using causal mathematical rules underscores that the universe is following causal rules. It is counter-productive to assign non-causality on nature to hide our deeper ignorance. However, the universe is a very complex and entangled system, depending upon the various ranges of the different forces. All the galaxies and the stars are influencing each other. The stars are holding the planets. Nevertheless, the ranges of the weak and the strong nuclear forces do not extend much beyond Femto meters.

The concept of entanglement in Quantum Mechanics (QM) without the necessity of some physical force of interaction between a set of entities arises due to one unnecessary assumption and one erroneous assumption in QM. First, let us underscore the unnecessary assumption. We have been trying to adhere to "wave particle duality" in interpreting superposition effects due to EM waves and particles. This "duality" arise only if we keep rejecting all possible causal interpretations. We have presented such a causal interpretation in sub-sections 4.1.3 for EM waves and 4.1.5 for particles. We just need to appreciate that there exist some causal interaction process consistent with our successful causal mathematics.

The erroneous assumption is due to our forceful attempt to make a single quantum entity exist simultaneously in two or its entire set of quantum mechanically allowed states at the same time. The hard causality demands that a well defined and a stable quantum entity, when excited, can exist in one and only one of its allowed set of excited states at a time. Only an ensemble of similarly prepared particles will have a population distribution over the entire set of allowed states. In fact, this is why Boltzmann's classical statistical relation has become so useful in representing the population distributions in QM also. Now, let us note that both the wave equations of Maxwell's and of Schrodinger's accept the linear superposition (sum) of all of their allowed solutions. This is the common origin of the Superposition Principle (SP) in classical and quantum physics. In classical optics, as per extended Huygens Principle of NIW, the physical meaning of classical SP is that the propagating waves can co-propagate and cross-propagate through the same volume at the same time without influencing each other's intrinsic propagation behavior in the absence of interacting material. However, particles do interact to alter their intrinsic identities in multiple possible ways, besides elastic and inelastic 
scattering. Since particles exist as space-finite entities, they normally cannot occupy precisely the same micro-volume either. Therefore, even though the particles executing various forms of harmonic oscillations to display phase properties, the NIW-property is not applicable for them. Therefore, physical process wise, the Schrodinger's SP is, different from Maxwell's SP. However, the causality must reign supreme in nature.

The early developers of QM have succeeded in initiating the culture that QM rigorously apply to a single particle, even though real experimental validations always require collecting data from an ensemble of similarly prepared system of particles. Howevere, they assumed that QM SP implicates that the same particle can exist in the normalized superposition of multiple allowed states. Consider the case of only two allowed states for a single particle, instead of $n$. This is introductory QM [17].

$$
\Psi=\psi_{1}+\psi_{2}=a_{1} \exp \left[i E_{1} t / \hbar\right]+a_{2} \exp \left[i E_{2} t / \hbar\right]
$$

Now, to make it valid for only one particle over all space $\Psi^{\bullet} \Psi$ must be normalized, meaning that $\int \Psi{ }^{\bullet} \Psi d x=1$

$$
\int \Psi \cdot \Psi d x=a_{1}^{2} \int\left|\psi_{1}\right|^{2} d x+a_{2}^{2} \int\left|\psi_{2}\right|^{2} d x+2 \operatorname{Re} a_{1} a_{2}^{*} \exp \left[i\left(E_{2}-E_{1}\right) t / \hbar\right] \int \psi_{1} \psi_{2}^{*} d x=1
$$

Then the mathematical logic proceeds as follows. Since both $\psi_{1}$ and $\psi_{2}$ represent the same single particle, they are already normalized; further $E_{2} \neq E_{1}$ to assure the existence of two distinct solutions (states). We now need to enforce the following two conditions to assure that $\Psi$ is normalized and the same single particle occupies both the states (entangled?):

$$
\left(a_{1}^{2}+a_{2}^{2}\right)=1 \text { and } \int \psi_{1} \psi_{2}^{*} d x=0
$$

The integral in Eq.5 implies that $\psi_{1}$ and $\psi_{2}$ should have been mathematically prepared as $\psi_{1} / a_{1}$ and $\psi_{2} / a_{2}$ to achieve the normalization (single-particle). This, I believe, is the approach in quantum computing when they write entangled states. However, the integral of Eq.5 is also worth pondering. It means that $\psi_{1}$ and $\psi_{2}$ are orthonormal to each other; they do not interfere or interact with each other. How can they? It would imply self-interference. The particle is in a superposition state in a magical, un-real world. Thus, Eq.4 is a mathematically correct construction, but does not represent physically valid interaction process in nature. Therefore, we should not give too much physics credence on the various interpretations generated out of these two mathematical equations. Let us compare Eq.1 and Eq.3. The "+" operator sign in Eq.1 is executed by a detector by virtue of the presence of polarizability $\chi$. There are no physical entity to execute the "+" operation in Eq.3. It is a correct and elegant mathematical statement without any reality in the physical world. In reality, if we can literally isolate one particle that can be excited to the allowed states of either $E_{1}$ or $E_{2}, E_{1} \neq E_{2}$ from the standpoint of energy conservation, it can never be in a superposition of both the states at the same instant because of conservation of energy. Accepting statistical ensemble interpretation of the quantum theory is a causal approach.

Similarly, the inequality condition of the elegant mathematical Bell's Theorem [18] has been formulated without any relevance to real physical experiment, without incorporating any interaction parameter with some experimental detector. It is elegant mathematics but without representing any causal physical interaction taking place in nature of nature.

Proper ensemble interpretation of actual quantum phenomena should be able to identify which interpretation of real quantum phenomena are worth perusing to extract better physics out of nature. QM formalism does represent individual atomic or other quantum phenomena. However, the basic QM formalism does not explicitly incorporate the incessant collisions of the QM entities under measurement due to the passing of a wide variety of EM radiation and cosmic rays. The finite lifetime of an excited state automatically implicates that it is sitting on a small energy-well in the energyspace, the depth of which determines the longevity in this state [19]. The statistically random collisions with the fluctuating background radiation can free it out of the mini-energy well to come down to a more stable lower state (level). This is the causal and physical reason behind the statistical behavior of excited quantum entities. In other words, quantum statistics is not a whole lot different from that for classical physics where there are $10^{23}$ molecules within a box of one cubic meter randomly colliding with each other when confined. We cannot write down all the equations for all possible degrees of freedom for $10^{23}$ molecules. For the QM world, it is worse. Unfortunately, the QM entities are in the open box of the entire universe; we can never box-out all the background fluctuations, neither have we been able to identify all background fluctuations. This is why, from the standpoint of measurement, the decisive detection of a single 
“click” can never help us validate any theory. Naturally, QM formalism aptly applies only to statistical ensemble average.

Let us consider an ensemble of stimulated quantum entities in one specific "upper state" that can come down multiple possible separate lower states when left alone. The background fluctuations will be stimulating each one of them randomly but differently to make the various possible downward transitions at different moments. In this physical picture, each one of them is conceptually identifiable, like the $10^{23}$ classical gas molecules in a meter-cubed box, due to the various different, but the specific stimulations they are experiencing. However, we cannot succeed in individually tracking them to identify which one is making which downward transition to which particular allowed lower state (of the allowed many), at least not with the existing technologies we have today. The key epistemological point is that we should not assume that an isolated single excited quantum entity remains in the normalized superposed states until the actual transition takes place. They do not reside in a single superposed state of "amplitude excitation". From the causality stand point, it is the statistically fluctuating background that triggers any specific transition.

\subsection{Classical Optics in view of NIW}

\subsubsection{Superposition Principle (SP) vs Superposition Effect (SE) in light of NIW}

Here we want to pay close attention to differentiate between observable parameters that we can measure and correlate with the theories in contrast to the parameters that are not directly measurable, albeit, referring to conceptually important parameters that have been necessary to develop the "correct" theory. For visible light, a.exp[i2 $\pi v t]$, the amplitude a is not directly observable, but $\mathrm{a}^{2}$ is, due to the quadratic response of detectors for high frequency EM waves, which is usually a quantum transition in the detecting molecule. In contrast, in the radio wave domain, the conduction electrons in a resonant LCR-circuit can directly respond to the incident oscillatory electromotive force generated across the LCRcircuit. An oscilloscope connected across the resistor in the LCR circuit can dynamically generate an oscillatory voltage signal that is proportional to the "amplitude" of the incident radio wave. Accordingly, when two or more waves are superposed on such a detector with broad resonance, the observable superposition effects are different for radio waves compared to the light waves, even though, the starting mathematical expression for the Superposition Principle (SP) would look identical. However, the expression for the measured Superposition Effect (SE) would be very different. This is one of the key reason for sustained confusion in interpreting the results of SE in the optical domain. The interpretational problem is even more compounded because; optical SE can be generated both (i) classically by a boundary layer of a beam combiner in an interferometer, and (ii) directly by a quantum detector [16].

\subsubsection{Superposition Effects in Diffraction Phenomenon in light of NIW}

Fresnel's mathematical representation of the Huygens Principle automatically incorporates the mathematical SP and NIW for EM waves, which is presented as the summation (integral) of all the Huygens' secondary wavelets. Young already demonstrated SE for light around 1802. Maxwell presented mathematical physics behind the SP around 1867 through his wave equation for EM waves. The wave equation accepts all linear combinations of harmonic oscillations as its solution. It is an inherent mathematical property of any wave equation. However, to generate the observable (measurable) SE, some physical entity must execute the physical operation of summing the independent amplitudes connected by the summation symbols. The energy transfer then takes place as the square of the resultant amplitude. The necessary critical epistemological discussions are missing in the current physics books even though mathematics is presented correctly. However, Huygens [4] explicitly mentions that his secondary wavelets evolves through each other without perturbing each other, or re-organizing the spatial and temporal energy distribution of each other. The physical meaning of Maxwell's wave equation is that the waves can co-propagate through each other keeping individual parametric values unperturbed in the absence of interacting materials. Thus, Maxwell's wave equation validates Huygens' Principle of secondary wavelets.

\subsubsection{Superposition Effects in Interference Phenomenon in light of NIW}

Because of the NIW property of EM waves, we should not be representing the superposition as direct summation of the waving field amplitudes as is customarily done in all books and literature. We need to apply the Interaction Process Mapping Epistemology (IPM-E) mentioned earlier. In this section, we will differentiate the generation of heterodyne beat signal due to the superposition of two waves with two different frequencies and underscore that observed SE differs based upon the detector's physics properties. 
Let us consider the case of superposition of two EM waves with closely similar but different frequencies [20]. When a frequency resonant detector can simultaneously respond to both the stimulating waves, the enforced oscillation of an LCR circuit, or of a photo detecting dipole, can execute the resultant amplitude oscillation. Therefore, the detector's physical response characteristics to the impinged fields determines the properties of the measurable (observable) data. First, consider two EM waves of closely similar frequencies, $v_{1}$ and $v_{2}$, in the radio frequency domain simultaneously stimulates a resonant LCR circuit, whose resonance breadth is wider than $\left(v_{1}-v_{2}\right)$. If the circuit response-figure is $\eta$, then one can measure an oscillatory current $i_{\text {total }}(t)$ through the resistor of the LCR circuit as a two-term Fourier sum-like current (Eq.6). The measurable current oscillates at a high frequency of $\left(v_{1}+v_{2}\right) / 2$, which is enveloped by a slower oscillatory current of $\left(v_{1}-v_{2}\right) / 2$. This is because the conduction electrons in the LCR circuit can directly respond to the resultant electromotive force (potential difference across the circuit) and follow the radio frequency. This is an AC current.

$$
i_{\text {total }}(t)=\eta E_{1}+\eta E_{2}=\eta a \cos 2 \pi v_{1} t+\eta a \cos 2 \pi v_{2} t=2 \eta a \cos 2 \pi \frac{v_{1}-v_{2}}{2} t \cdot \cos 2 \pi \frac{v_{1}+v_{2}}{2} t
$$

However, if the EM wave is in the optical frequency domain, the detectors that can respond to this high frequency are quantum detectors that are atomic, molecular or their assembly, through quantum level transition. Such quantum dipolar excitations are normally expressed as complex functions, $\psi_{1,2} \equiv \chi a \exp \left[-i 2 \pi v_{1,2} t\right]$, where $\chi$ is the linear dipolar polarizability of the quantum detector's dipole element. Here also we are assuming that the resonant response breadth of the dipole is wider than $\left(v_{1}-v_{2}\right)$ around the mean frequency. The observed photoelectric current is oscillatory at the

$$
D(t)=\Psi * \Psi=\left|\psi_{1}+\psi_{2}\right|^{2}=\left|\chi a e^{-i 2 \pi v_{1} t}+\chi a \mathrm{e}^{-i 2 \pi v_{2} t}\right|^{2}=2 \chi^{2} a^{2}\left[1+\cos 2 \pi\left(v_{1}-v_{2}\right) t\right]
$$

difference frequency only (Eq.7). However, it is a DC current, not an AC current as it is for the radio wave and the LCRcircuit detector. This is because, inside a photo detector with broad energy bands, electrons are undergoing one-way upper level transition at this oscillatory rate and an externally applied voltage draws them out through an external LCR circuit as the oscillatory DC current. Of course, to display faithfully this oscillatory current, the response time constant of the external circuit of the photo detector electronics must be faster than the optical difference frequency $\left(v_{1}-v_{2}\right)$. If it is much slower, the photo current will again be a steady DC current proportional to the sum total intensity of the waves, $2 \chi^{2} a^{2}$. We just need to recognize that our math must faithfully model the physical interaction processes that generate the measurable data. Only an appropriate responsive physical detector can execute the operation "+" in the above equations and its response characteristics determine the measurable superposition effect.

For the case of optical quantum detector, one can use real, instead of complex mathematical representation. However, the mathematical steps to derive the final result require taking a time averaging integration over a couple of cycles to derive the same result, as is in Eq.8. Nature's logics do not really follow human invented math, especially, the complex representation.

$$
D(t)=\frac{1}{T} \int_{t}^{t+T}\left[\chi E_{1}+\chi E_{2}\right]^{2} d t=\frac{1}{T} \int_{t}^{t+T}\left[\chi a \cos 2 \pi v_{1} t+\chi a \cos 2 \pi v_{2} t\right]^{2} d t \approx 2 \chi^{2} a^{2}\left[1+\cos 2 \pi\left(v_{1}-v_{2}\right) t\right]
$$

Then the physical meaning of Eq.8, most likely, is that a quantum detector does not undergo any instantaneous quantum transition. It needs a couple cycles-equivalent time period to assess whether the stimulating signal is resonant for its quantum transition. We may call it a "quantum compatibility sensing period" [20].

\subsubsection{Superposition Effects in Spectrometry}

Classical formulation of spectrometer response function is derived by propagating a Fourier monochromatic mode through the spectrometer. Such a signal cannot exist in nature, as that would violate the law of conservation of energy. Besides, the present classical formulation misses several important physical characteristics of spectrometers. We have derived [Ch.5 in 3] a causal formulation of spectrometer response function by propagating a finite classical light pulse 
with a single carrier frequency and of finite width, $\Delta \mathrm{t}$ to obtain a generic spectrometer pulse-response function. Under the condition of $\Delta \mathrm{t}>\tau_{0}$, where $\tau_{0}=\mathrm{N} \tau$. Here $\tau=\mathrm{m} \lambda / \mathrm{c}$ is the spectrometer step delay and $\mathrm{N}$ is number of actual superposed beams on the detector; $\mathrm{m}$ is the order of diffraction (for gratings), or the order of interference for Fabry-Perots. Note also that, by virtue of NIW, simple superposition of Fourier amplitude modes cannot generate new re-distribution of wave energy. Only a quadratic process can create energy re-distribution. These causal facts should be properly underscored to avoid any confusion in interpreting physical E-vector oscillation frequency content vs the spectrometer broadened impulse function. Further, spectrometers being memoryless linear optical beam replicators, they cannot carry out complex mathematical Fourier transform algorithms to determine the Fourier monochromatic frequencies that could be mathematically contained in a pulse envelope.

\subsubsection{Superposition Effects in Coherence Phenomenon in light of NIW}

Traditionally, we define the degree of coherence, or the visibility of fringes, for any light beam through an autocorrelation integral. The integrated energy, detected due to the superposition of a pair of replicated and temporally translated pulses, provides the measured value of the degree of coherence. This well-defined approach to characterize light beams has been extremely valuable. In reality, the joint stimulation of a quadratic response of a detector due to the distribution of different amplitudes and different phases determine the recorded fringe visibility. Therefore, the fringe visibility is a joint property of a quadratic detector and the amplitude/phase distribution in the light beam. So, the measured fringe visibility should not be characterized as the coherence property of the light beam alone.

We should note that neither a linear optical system, nor a simple quadratic optical detector without any built-in memory, could execute Fourier transform algorithm. However, in the traditional coherence theory, mathematical Fourier frequency and the source generated physical frequencies are used in mathematical formalism interchangeably. We have underscored this logical inconsistency in Ch.6 of ref. 3.

\subsubsection{Superposition Effects in Laser Mode-Lock Phenomenon in light of NIW}

The traditional books on optics and lasers present the mode-locking phenomenon as direct sum of cavity longitudinal mode amplitudes and the required temporal phases are stabilized by the intra-cavity mode-locker. The implication is that a Fourier summation is executed by the wave amplitudes themselves within the cavity to generate the temporal oscillation of the cavity energy. By virtue of the NIW-property of waves, this approach fails to represent the deeper active roles that are played by the intra-cavity mode-locking materials. The actual energy reorganization happens due to the cyclic temporal-gating property of the intra-cavity phase-locking devices, which is a quadratic process. As per Fourier theory, all the in-phase longitudinal modes should collapse into a single central optical frequency in the output. However, we already know the various engineering use of the frequency comb (all the longitudinal cavity modes) contained in each of the pulses coming out of mode-locked lasers [Ch.7 in 3].

\subsubsection{Superposition Effects in Material Dispersion Phenomenon in light of NIW}

The group velocity has been one of the key property taught through every books on material dispersion. The derivation is by summing two phase-steady CW waves with two different frequencies. This is again counter to the NIW-property of waves. While the two waves are co-propagating with parallel Poynting vectors, they still preserve their independent oscillations. This is why wavelength multiplexed signals can be sent through many kilometers of single mode fiber and fully recovered by a spectrometer at the output. Thus, the concept of group velocity is not a good physical parameter to characterize multi-frequency wave propagation [Ch.8 in 3].

\subsubsection{Superposition Effects in Polarization Phenomenon in light of NIW}

We are taught that light beams of orthogonal polarization do not interfere. However, we have started this paper by underscoring the NIW-property of all waves, whether polarized orthogonal or parallel. It is the dipolar properties of dielectric material boundaries and detecting molecules whose joint oscillatory response characteristic is restricted to only one polarizable axis in isotropic media at a time. Anisotropic media show two orthogonally fixed directions of polarizability and consequent behavior. Thus, the response of material to multiple incident waves of different polarization will be always resolved into one or the other axial vibration. For an isotropic detector, receiving multiple polarized beams, it will first accept amplitude stimulation from the multiple waves as their vectorial cosine projections, and then absorb energy proportional to the square modulus of the vectorial sum of the projected amplitudes [Ch.9 in 3]. This is congruent with the analysis presented by Jones' matrix method. 


\section{LESSONS LEARNED - ADVANCING OUR EPISTEMOLOGY}

\subsection{Causality is at the core of understanding nature}

Physicists are rightly excited about their collective accomplishments accumulated just over the last four hindered years. Today we understand the basic behaviors of both the world of elementary particles in the micro domain and the world of galaxies in the macro domain. This is a staggeringly accelerated accumulation of knowledge, even though our ancestors first started walking upright almost five million years ago. The two core reasons [Ch.12 in 3] are the philosophy of accepting only the (i) reproducible evidence based science, and (ii) creating mathematical theories equating cause-effect relationship, which validate the measured evidences. However, mathematics played a significantly stronger role in facilitating the acceleration while validating our evidence based knowledge, as if they were the "God's Equations" [21]. Unfortunately, this belief has corrupted our belief in hard causality, even though we construct all of our working theories to equate measurable effect (evidence) with the postulated cause behind the emergence of the effect. For example, mathematical Fourier modes exists in all space. Hence, it cannot represent any real physical signal, as that would violate the law of conservation of energy. Yet, we tell nature when and where to violate causality using Heisenberg's Uncertainty Principle. Note that this mathematical "uncertainty" relation is derived by using the ad hoc multiplication of the half-widths of a pair of conjugate Fourier transform functions and mathematically justifying its validity of greater than unity using Schwartz inequality. Surprisingly, QM formalism consistently does excellent in validating observations without the need to be propped up by this uncertainty relation. Our understanding of physics is not seriously hindered by our inability to measure simultaneously multiple physical parameters of a single physical entity using the same experimental set up. We rarely do that even in classical physics.

All theories are limited theories since they are always constructed based upon our limited knowledge of the integrated universe. Therefore, fundamental postulates of all working theories must have captured some ontological truth out of nature. To isolate out that these ontological components, we need to unify different working theories into a new broader working theory just as Maxwell unified Electrostatics and Magnetostatics into Electromagnetism. So far, it has remained almost invincible since 1867. The prevailing culture of systematically controlling the challenges to the theories of Relativity and Quantum Mechanics is thwarting the progress in physics and is alienating many brilliant younger physicists to choose different professions [2].

\subsection{Insufficiency of Measurable Data Modeling Epistemology (Incorporate Interaction Process Mapping Epistemology, or IPM-E)}

The history of the evolution of organized knowledge by humans clearly demonstrate that they must keep advancing in steps by recognizing mistakes and implementing refinements through corrections. Accordingly, we can safely assume that the current "working" theories of Classical, Relativity and Quantum Physics will also undergo changes and refinements to get closer to ontological reality of nature. These working theories have not yet laid the final foundation of the edifice of physics.

No measured data about a physical entity, collected through interaction with a reference entity, can give us complete information about our target entity. This is because, in the real world of today, we do not know complete information about any physical entity. This is a fundamental Information Retrieval Problem (IRP) at the current state of evolution of the human species. We would not be able to overcome this IRP in the near future. However, we can still iteratively advance our successes if we properly focus our attention to the real physical interaction processes that nature executes to generate measurable data in our instruments. These interaction processes embody the true ontological reality; but they are beyond our direct access, because we cannot peer into the micro world directly. This inaccessible information should not be lumped away behind Uncertainty Principle or similar postulates, even when we can construct supporting mathematical theorems. However, we can imagine, visualize, mathematically model and then keep on iteratively advancing towards the ontological reality. We have defined this approach as the incorporation of Interaction Process Mapping Epistemology, or IPM-E [Ch.12 in 3]. A simple example can illustrate the point. We have ushered in the Knowledge Age by the building Global Internet system. The underlying technology consists of utilizing four engineering processes on electrons and photons - (i) generation, (ii) modulation, (iii) propagation and (iv) detection. However, we still do not understand with certainty what are electrons and photons built out of. 


\section{3. “Unreasonable Power of Mathematics" [22] and its limitations}

Mathematics is the best logical language invented by humans. However, there are many mathematical logics (theorems. lemmas, etc.), which have nothing to do with the rules of operation going on in the perpetually evolving universe. Further, we do not yet know all there is to know about the real working rules behind the entire universe. We are still wandering around in determining the best set of educated-hypotheses to put together the evidence-based puzzle pieces in our iterative attempts to construct the separate sub-segments of the entire cosmic map. The integral cosmic map is still lying in front of us un-solved. We have no other option but to advance in piece-meal, in iterative steps, where the individual working theories are just segmented, disjoint map pieces. All the different working rules that solve the submaps, cannot be simply added together to solve the grand cosmic map. We already know that we cannot derive the emergent behavior of very complex system using the rules that work very well for the constituent elementary subsystems. We are computing the molecular behaviors predictably using the successful models out of QM. However, we are nowhere near to derive the living behaviors of the biological world out of the DNA molecules, as yet. Neither, we have been successful in developing an acceptable unified field theory. However, we are progressing steadily through iterative steps without seriously accepting any working model as the "final" model like Relativity or Quantum Mechanics.

We need to incorporate "Reality Ontology" (RO) on our working mathematical formalism by demanding: (i) one-toone correspondence between each mathematical symbol and an actual state of an entity in nature and (ii) one-to-one correspondence between each mathematical operator and the theory-allowed interaction processes between the states of the adjoining entities (mathematical symbols). By demanding such a "process driven interpretation" for each and every mathematical symbol and operator in a theory, we will be able to discover the power and the beauty behind its success, as well as its limits.

Let us recall again Eq.1 that represents our reality-ontology approach to understanding Superposition Principle as a physical process facilitated by detecting dipole of linear polarizability parameter $\chi$ while being stimulated by two electric fields.

$$
\begin{gathered}
\psi=\chi E_{1}+\chi E_{2}=\chi a_{1} e^{i 2 \pi v(t+\tau)}+\chi a_{2} e^{i 2 \pi v t} \\
E_{\text {res. }}=E_{1}+E_{2}=a_{1} e^{i 2 \pi v(t+\tau)}+a_{2} e^{i 2 \pi v t}
\end{gathered}
$$

We have re-written Eq.9 without the interaction-strength parameter $\chi$. This is the traditional style of writing the Superposition Principle without any reference to which physical entity is executing the summation operation "+" inserted between $E_{1} \& E_{2}$. Being solutions of the Maxwell's wave equation, linear EM waves, $E_{1} \& E_{2}$, follow the NIW property; they cannot sum themselves. Hence, Eq.9 does not represent any physical process. It is a self-consistent mathematical statement only. Two waves are propagating through the same spatial volume. Physics equations must attempt to model ontological interaction processes in nature. Representation of Eq.9 has generated many mistakes in interpretations of many optical phenomena, both classical and quantum, which are still continuing, as we have summarized in this paper.

Accordingly, we should pay close attention to apply Interaction Process Mapping Epistemology (IPM-E) in constructing mathematical formalism to represent interaction processes in nature. Reductionism through judicial use of physical parameters and mathematical operators representing interaction causes (e.g.. r.h.s. of Eq.1) and synthesis ("emergence") through equating with the resultant measurable outcome have to advance hand-in-hand (l.h.s. of Eq.1)

\section{CONCLUSIONS}

During the three centuries, from middle of 1600 through the end of 1800 , development and use of science and engineering of light led the foundational development of Physics [23]. Innumerable scientists contributed to this cultural shift in science - Galileo, Newton, Huygens, Young, Fresnel, Maxwell, etc., etc. Huygens' ether concept was given an enduring mathematical credence by Maxwell after he showed that the velocity of light is determined by the physical stress-related properties of space $\varepsilon_{0} \& \mu_{0}$, as $c^{2}=1 / \varepsilon_{0} \mu_{0}$. Then, Einstein's $m_{0}=E / c^{2}=E \varepsilon_{0} \mu_{0}$ clearly established the deeper electromagnetic nature of matter. Inertia is an emergent property of particles built out of localized oscillations of the same universal Complex Tension Field, or CTF [3,6]. Accordingly, optical scientists and engineers, who are constantly dealing with electromagnetism, should pick up the leadership role to re-initiate the advancement of Physics out of its current stagnant state [1,2,3,22]. 


\section{REFERENCES}

[1] L. Smolin, [The Trouble with Physics: The Rise of String Theory, The Fall of a Science, and What Comes Next], Houghton Mifflin Co., (2007).

[2] S. Hossenfelder, [Lost in Math: How Beauty Leads Physics Astray], Hachette Book Group (2019).

[3] C. Roychoudhuri, [Causal Physics: Photon by Non-Interaction of Waves], Taylor and Francis (2014).

[4] C. Huygens, [Treatise on Light], drafted in 1678. Project Gutenberg edition, http://www.gutenberg.org/ebooks/14725 (2005).

[5] M. Born and E. Wolf, [Principles of Optics], Cambridge U. Press, 6 ${ }^{\text {th }}$ Ed., (1980).

[6] C. Roychoudhuri, "Next frontier in physics - space as a complex tension field”, J. of Mod. Physics, (2012).

[7] C. Roychoudhuri, "Locality of Superposition Principle L. is dictated by detection process", Phys. Essays, 19 (3), (2006).

[8] C. Roychoudhuri, "Urgency of evolution process congruent thinking in physics. An advocacy to elevate the prevailing abstract physics-thinking towards a functionally useful reverse-engineering thinking”, Proc. SPIE 9570 (2015).

[9] C. Roychoudhuri and M. Ambroselli, "Can one distinguish between Doppler shifts due to source-only and detectoronly velocities?”, Proc. SPIE 8832-49 (2013).

[10] C. Roychoudhuri, “Could space be considered as the inertial rest frame?”, Proc. SPIE Vol.9570-30 (2015).

[11] A. Einstein, [Relativity: The Special and General Theory], Three River Press, (1961).

[12] C. Roychoudhuri, "Consequences of repeated discovery and benign neglect of Non-Interaction of Waves (NIW)", Proc. SPIE Vol. 104521045215 (2017). Free download site: https://spie.org/Publications/Proceedings/Paper/10.1117/12.2266216 (2017)

[13] W. Demtroder, “Laser Spectroscopy: Basic Concepts and Instrumentation,” 2nd ed., Springer-Verlag, (1998).

[14]C. Roychoudhuri and N. Prasad, "Complex interaction processes we need to visualize that successfully fill the quantum cup of a detector”, Proc. SPIE Vol.10926, 109260T (2019). doi: 10.1117/12.2514835. (2019).

[15] G. Grynberg, A. Aspect and C. Fabre, [Intro uction to Quantum Optics: From the Semi-Classical Approach to Quantized Light], Cambridge U. Press, (2010).

[16] CR SPIE? C. Roychoudhuri and N. Prasad, "Complex interaction processes we need to visualize that successfully fill the quantum cup of a detector”, Proc. SPIE Vol.10926, 109260T (2019). doi: 10.1117/12.2514835. (2019).

[17] H. A. Kramer, [Quantum Mechanics], Dover Publication, (1964).

[18] J. S. Bell, [Speakable and Unspeakable in Quantum Mechanics: Collected Papers on Quantum Philosophy], Cambridge U. Press, (2004)

[19] C. Roychoudhuri, "Can photo sensors help us understand the intrinsic difference between quantum and classical statistical behavior?”, AIP Conf, Proc. Vol.1101, pp.167-177, (2009).

[20] M. Ambroselli, P. Poulos and C. Roychoudhuri, [Nature of EM waves as observed \& reported by detectors for radio, visible and gamma frequencies], Proc. SPIE, Vol.8121-41 (2011).

[21] A. D. Aczel, [God's Equation: Einstein, Relativity, and the Expanding Universe], Delta, Random House, (2000).

[22] E. P. Wigner, "The unreasonable effectiveness of mathematics in the natural sciences", Communications on Pure and Applied Mathematics, Vol.13 XIII, pp.1-14, (1960).

[23] V. Ronchi, [The Nature of Light], Harvard U. Press, (1970). 